ANNALES

POLONICI MATHEMATICI

$90.3(2007)$

\title{
A note on the Nullstellensatz for c-holomorphic functions
}

\author{
by Maciej P. Denkowski (Kraków and Bordeaux)
}

\begin{abstract}
We begin this article with a graph theorem and a kind of Nullstellensatz for weakly holomorphic functions. This yields a general Nullstellensatz for c-holomorphic functions on locally irreducible sets. In Section 2 some methods of Płoski-Tworzewski permit us to prove an effective Nullstellensatz for c-holomorphic functions in the case of a proper intersection with the degree of the intersection cycle as exponent. We also extend this result to the case of isolated improper intersection, generalizing a result of E. Cygan. The last section is devoted to some considerations on the dimension of the zero-sets of c-holomorphic mappings.
\end{abstract}

1. Introduction. Let $A \subset \Omega$ be an analytic set in an open set $\Omega \subset \mathbb{C}^{m}$. Recall (see [R] where this notion was first introduced and also [€], [Wh]) that a function $f: A \rightarrow \mathbb{C}$ is called $c$-holomorphic if it is continuous and the restriction of $f$ to the subset $\operatorname{Reg} A$ of regular points is holomorphic. We denote by $\mathcal{O}_{\mathrm{c}}(A)$ the ring of c-holomorphic functions on $A$. A mapping is cholomorphic iff all its components are c-holomorphic functions. The following theorem is fundamental for all we shall do (cf. [Wh, 4.5Q]):

THEOREM 1.1. A mapping $f: A \rightarrow \mathbb{C}^{n}$ is c-holomorphic iff it is continuous and its graph $\Gamma_{f}:=\{(x, f(x)) \mid x \in A\}$ is an analytic subset of $\Omega \times \mathbb{C}^{n}$.

For a more detailed list of basic properties of c-holomorphic mappings see [Wh], [D]. The notion of weakly holomorphic functions (H. Cartan) is much better known. We just recall that it refers to functions defined and holomorphic on $\operatorname{Reg} A$ and locally bounded near the singularities (i.e. on the whole of $A$ ). A mapping is called weakly holomorphic if all its components are weakly holomorphic. We denote by $\mathcal{O}_{\mathrm{w}}\left(A, \mathbb{C}^{n}\right)$ the ring of weakly holomorphic mappings and put $\mathcal{O}_{\mathrm{w}}(A):=\mathcal{O}_{\mathrm{w}}(A, \mathbb{C})$. More details can be found in [Wh].

2000 Mathematics Subject Classification: 32B15, 32A17.

Key words and phrases: complex analytic sets, c-holomorphic and weakly holomorphic mappings, Nullstellensatz, intersection theory. 
We just recall that if $A=\bigcup A_{\iota}$ is the decomposition of $A$ into irreducible components, then a weakly holomorphic function $f$ has a unique extension onto $\operatorname{Reg} A_{\iota}$ for each $\iota$ (and that works in fact for germs). Thus $f$ is weakly holomorphic iff it is so on each irreducible component of $A$. Over a singular point $a \in \operatorname{Sng} A$ a weakly holomorphic function is multi-valued and takes at most as many values as the number of irreducible components of the germ $A_{a}$.

It may be useful to state explicitly what we mean by proper intersection. Let $X, Y$ be analytic sets in $\Omega \subset \mathbb{C}^{m}$ of pure dimensions $p$ and $q$ respectively. The intersection $X \cap Y$ is called proper if it has pure dimension $p+q-m$, i.e. at each intersection point the dimension is the minimal possible. In the opposite case the intersection is said to be improper.

For the definition and properties of the intersection cycle $X \cdot Y$ we refer the reader to $[\mathrm{Dr}]$ (for the proper case) and [T] (for the general case, see also [ATW] for isolated improper intersection). We just recall that if $X \cap Y=\bigcup S_{\iota}$ is the (locally finite) decomposition into irreducible components, then the intersection cycle is the formal sum

$$
X \cdot Y=\sum i\left(X \cdot Y, S_{\iota}\right) S_{\iota},
$$

where $i\left(X \cdot Y, S_{\iota}\right)$ is the intersection multiplicity of $X \cap Y$ along the component $S_{\iota}$ computed following [Dr] or [T].

The degree of the cycle $X \cdot Y$ at a point $a \in X \cap Y$ is the number

$$
\operatorname{deg}_{a}(X \cdot Y):=\sum i\left(X \cdot Y, S_{\iota}\right) \operatorname{deg}_{a} S_{\iota},
$$

where $\operatorname{deg}_{a} S_{\iota}$ stands for the classical degree of the analytic set $S_{\iota}$ at $a$ (with the convention that $\operatorname{deg}_{a} S_{\iota}=0$ if $a \notin S_{\iota}$; thus the above sum is finite).

Finally, let $U \subset \mathbb{C}^{k}$ be open and connected. If $\varphi: A \rightarrow U$ is a proper c-holomorphic mapping and $A$ has pure dimension $k$, then $\pi: \Gamma_{\varphi} \rightarrow U$, where $\pi: \mathbb{C}^{m} \times \mathbb{C}^{k} \rightarrow \mathbb{C}^{k}$ is the natural projection, is a branched covering (see e.g. [Dr], [Wh] or [€] ) and so has finite multiplicity (or covering number, see e.g. [Dr]). We call it the multiplicity of $\varphi$.

\section{General Nullstellensatz for weakly holomorphic functions.} Let $A$ be an analytic subset of some open set $\Omega \subset \mathbb{C}^{m}$.

We begin with a useful and apparently not known graph theorem for weakly holomorphic functions. It is a weakly holomorphic counterpart of Theorem 1.1. Recall that an analytically constructible subset of $\Omega$ is a set which can be written locally in $\Omega$ as $\bigcup_{\iota=1}^{p} \bigcap_{j=1}^{q_{j}}\left\{F_{\iota j} *_{\iota j} 0\right\}$, where $*_{\iota j} \in\{=, \neq\}$ and $F_{\iota j}$ are holomorphic (see [€]).

Theorem 2.1. Let $f: \operatorname{Reg} A \rightarrow \mathbb{C}^{n}$ be a continuous mapping locally bounded on $A$. The following three conditions are then equivalent: 
(1) $f \in \mathcal{O}_{\mathrm{w}}\left(A, \mathbb{C}^{n}\right)$.

(2) The closure $\bar{\Gamma}_{f}$ is an analytic subset of $\Omega \times \mathbb{C}^{n}$.

(3) $\Gamma_{f}$ is analytically constructible in $\Omega \times \mathbb{C}^{n}$.

Proof. First note that we may restrict ourselves to the case $n=1$ since $\Gamma_{f}=\bigcap_{j=1}^{n} \Gamma_{j}$, where

$$
\Gamma_{j}:=\left\{\left(x, y_{1}, \ldots, y_{j-1}, f_{j}(x), y_{j+1}, \ldots, y_{n}\right) \mid x \in \operatorname{Reg} A, y_{\iota} \in \mathbb{C}\right\} .
$$

We may as well assume that $A$ has pure dimension $k$ (using restrictions to the irreducible components of $A$ ) with $0<k<m$ (otherwise, since there are no singularities, there is nothing to do-cf. the analytic graph theorem).

If we have $(1) \Leftrightarrow(2)$, the equivalence $(2) \Leftrightarrow(3)$ is quite immediate. Indeed, if $\bar{\Gamma}_{f}$ is analytic, then $\Gamma_{f}=\bar{\Gamma}_{f} \backslash(\operatorname{Sng} A \times \mathbb{C})$ is the difference of two analytic sets, and hence is analytically constructible. On the other hand, if $\Gamma_{f}$ is analytically constructible, then its closure is analytic and so $f \in \mathcal{O}_{\mathrm{w}}(A)$.

We now turn to proving $(1) \Leftrightarrow(2)$. If $\bar{\Gamma}_{f}$ is analytic, then so is $\Gamma_{f}$ in $(\Omega \backslash \operatorname{Sng} A) \times \mathbb{C}$. Thus by the analytic graph theorem, $f$ is holomorphic on $\operatorname{Reg} A$. Since by assumption it is locally bounded on $A$, we have $f \in \mathcal{O}_{\mathrm{w}}(A)$.

Now suppose that $f \in \mathcal{O}_{\mathrm{w}}(A)$. The problem being local, we may assume that $h \in \mathcal{O}(\Omega)$ is a global universal denominator for $A$ (cf. Oka's theorem, see [ $€])$. Then we can find $g \in \mathcal{O}(\Omega)$ such that $f h=g$ on Reg $A$. Consider now the analytic set $X:=\{(z, t) \in A \times \mathbb{C} \mid h(z) t=g(z)\}$. It remains to observe that the set

$$
\begin{aligned}
\Gamma_{f} \cap\{(z, t) & \in \Omega \times \mathbb{C} \mid h(z) \neq 0\} \\
& =X \cap(\operatorname{Reg} A \times \mathbb{C}) \cap\{(z, t) \in \Omega \times \mathbb{C} \mid h(z) \neq 0\} \\
& =X \backslash[(X \cap(\operatorname{Sng} A \times \mathbb{C})) \cup\{(z, t) \in \Omega \times \mathbb{C} \mid h(z)=0\}]
\end{aligned}
$$

is dense in $\Gamma_{f}$. Its closure in $\Omega \times \mathbb{C}$ is clearly analytic.

Note also that by this theorem the zero-set of a weakly holomorphic function is analytically constructible and so its closure is analytic. It is more natural, however, to consider the intersection of the closure of the graph with $A \times\{0\}$ as a substitute for the zero-set of a weakly holomorphic function.

Now we turn to proving the general weakly holomorphic Nullstellensatz.

THEOREM 2.2. Let $A \subset \mathbb{C}^{m}$ be an analytic germ at zero and suppose that $g, f_{1}, \ldots, f_{n} \in \mathcal{O}_{\mathrm{w}}(A)$ are such that

(1) $0 \in \bar{\Gamma}_{f}$, where $f:=\left(f_{1}, \ldots, f_{n}\right)$,

(2) $(x, 0) \in \bar{\Gamma}_{f} \cap\left(A \times\{0\}^{n}\right)$ implies $\bar{\Gamma}_{g} \cap(\{x\} \times \mathbb{C})=\{(x, 0)\}$ for all $x$ in some neighbourhood of zero. 
Then there exists an integer $p \geq 1$ such that on $\operatorname{Reg} A$,

$$
g^{p}=\sum_{j=1}^{n} h_{j} f_{j}
$$

for some $h_{j} \in \mathcal{O}_{\mathrm{w}}(A), j=1, \ldots, n$.

Proof. Let $\varrho: N \rightarrow A$ be a local normalization of $A$ at zero (we consider $A$ as an analytic subset of some neighbourhood of zero). Then $\widetilde{g}:=g \circ \varrho$ and $\tilde{f}_{j}:=f_{j} \circ \varrho$ are holomorphic on the normal germ $N$.

Observe now that $\widetilde{g}^{-1}(0) \supset \bigcap_{j} \tilde{f}_{j}^{-1}(0)$. Indeed, if $\tilde{f}_{j}(x)=0$ for all $j$, then either $\varrho(x) \in \operatorname{Reg} A$, in which case $\varrho(x) \in \bigcap_{j} f^{-1}(0)$ and so $\widetilde{g}(x)=0$, or $\varrho(x) \in \operatorname{Sng} A$. If the latter occurs, then we take a sequence of points $\operatorname{Reg} A \ni a_{\nu} \rightarrow \varrho(x)$. The sequence $x_{\nu}:=\varrho^{-1}\left(a_{\nu}\right)$ has a subsequence $\left\{x_{\nu_{\mu}}\right\}$ converging to $x$ (by the properness of $\varrho$ ). Then $f_{j}\left(a_{\nu_{\mu}}\right) \rightarrow 0$ for all $j$ and so $(\varrho(x), 0) \in \bar{\Gamma}_{f}$. But then $g\left(a_{\nu_{\mu}}\right) \rightarrow 0$, whence $\widetilde{g}(x)=0$.

Now we apply the holomorphic Nullstellensatz to $\widetilde{g}, \widetilde{f}_{1}, \ldots, \widetilde{f}_{n}$ (see [€]) obtaining holomorphic functions $\widetilde{h}_{j}$ such that for some $p \geq 1$,

$$
\widetilde{g}^{p}=\sum_{j=1}^{n} \widetilde{h}_{j} \widetilde{f}_{j}
$$

in a neighbourhood of the fibre $\varrho^{-1}(0)$. For simplicity assume for the time being that this holds on the whole of $N$ (in any case the matter is local).

Define $h_{j}(a):=\widetilde{h}_{j}\left(\varrho^{-1}(a)\right)$ for $a \in \operatorname{Reg} A$. In this way we clearly get some holomorphic functions $h_{j}: \operatorname{Reg} A \rightarrow \mathbb{C}$. But $\varrho$ being proper, all $h_{j}$ are bounded near the singularities and so they are weakly holomorphic.

Indeed, fix $j$ and take any point $a \in \operatorname{Sng} A$. For an arbitrarily small compact neighbourhood $U$ of $a$ the set $V:=\varrho^{-1}(U)$ is compact and so $\left|\widetilde{h}_{j}\right|$ is bounded on it. If the $\left|h_{j}\right|$ were unbounded on any such neighbourhood $U \backslash \operatorname{Sng} A$, we would find a sequence $\operatorname{Reg} A \ni a_{\nu} \rightarrow a$ such that $\left|h_{j}\left(a_{\nu}\right)\right| \rightarrow \infty$. However, $h_{j}\left(a_{\nu}\right)=\widetilde{h}_{j}\left(\varrho^{-1}\left(a_{\nu}\right)\right)$ and so it cannot be unbounded.

To finish the proof we just observe that (\#) remains true if we omit the tildes and restrict ourselves to a neighbourhood of zero intersected with $\operatorname{Reg} A$. To be more precise, as a neighbourhood in which (\#) holds we may take an open set $U$ of the form $\bigcup_{j=1}^{r} U_{j}$, where $r$ is the number of points in the fibre $\varrho^{-1}(0)$ and the union is disjoint. We may also ask that for any $j, U_{j}$ be connected. Clearly, $V:=\varrho(U)$ is an open neighbourhood of zero in $A$. Then on $V \backslash \operatorname{Sng} A$ the mapping $\varrho$ is invertible and its inverse sends a connected neighbourhood of $a \in V \backslash \operatorname{Sng} A$ (contained in $V \backslash \operatorname{Sng} A$ ) into exactly one of the $U_{j}$. There we have (\#) which becomes the required formula in the neighbourhood of $a$ after composition with $\varrho^{-1}$. 
EXAmple 2.3. Consider Whitney's umbrella $A:=\left\{x^{2} y=z^{2}\right\} \subset \mathbb{C}^{3}$ and the weakly holomorphic function $g(x, y, z)=z / x$ defined on it. We have Sng $A=\{0\} \times \mathbb{C} \times\{0\}$ and $g$ has two possible values over any point of Sng $A \backslash\{0\}^{3}$, namely $\sqrt{y}$ and $-\sqrt{y}$ (properly understood in the complex sense). On the other hand, $g(0)$ is well defined and equals zero. In particular $\bar{\Gamma}_{g} \cap(A \times\{0\})=\{0\}^{4}$.

Consider now $f_{1}(x, y, z)=z$ and $f_{2}(x, y, z)=x-y$ on $A$. The only common zero of these functions is the origin. They satisfy the assumptions of our theorem and indeed

$$
g^{3}=f_{1}-g f_{2} .
$$

A somewhat less interesting example may be given using the functions $f_{1}(x, y, z)=x$ and $f_{2}(x, y, z)=y$ on $A$. In this case $g^{2}$ is just $f_{2}$.

EXAMPLE 2.4. The assumption of univalence of $g$ over the zeroes of $f$ (i.e. over the set $\left.\bar{\Gamma}_{f} \cap\left(A \times\{0\}^{n}\right)\right)$ is essential. To see this, consider the set $A:=\{x y=0\}$ in $\mathbb{C}^{2}$ and, on $\operatorname{Reg} A$, the functions $f(x, y)=x-y$ and $g(x, 0)=x^{2}($ if $x \neq 0), g(0, y)=1$ (if $\left.y \neq 0\right)$. Both are weakly holomorphic on $A$ and

$$
f^{-1}(0)=\{0\}^{2}=\bar{\Gamma}_{g} \cap(A \times\{0\}),
$$

while $\bar{\Gamma}_{g} \cap(A \times \mathbb{C})=\{(0,0),(0,1)\}$. A straightforward computation shows that within the class of weakly holomorphic functions, no power of $g$ can be divisible by $f$ in any neighbourhood of zero. If $g^{p}=h f$ for some $p \in \mathbb{N}$, then $|h|$ must be unbounded near zero on the component $\{x=0\} \subset A$.

We derive from the theorem above a general c-holomorphic Nullstellensatz on locally irreducible analytic sets.

Corollary 2.5. Let $A$ be a locally irreducible analytic set and suppose that $g, f_{1}, \ldots, f_{k} \in \mathcal{O}_{\mathrm{c}}(A)$ are such that $g^{-1}(0) \supset \bigcap_{j} f_{j}^{-1}(0)$. Then for each $a \in \bigcap_{j} f_{j}^{-1}(0)$ there is an integer $p \geq 1$ and some c-holomorphic functions $h_{j}$ in a neighbourhood of a such that

$$
g^{p}=\sum_{j=1}^{k} h_{j} f_{j}
$$

in this neighbourhood.

Proof. For simplicity let $a=0$. The set $A$ being locally irreducible, we have $\mathcal{O}_{\mathrm{c}}(A)=\mathcal{O}_{\mathrm{w}}(A)$. We now apply the preceding theorem, since our assumptions imply both hypotheses (1) and (2). The assertion follows by continuity or by applying the identity principle as in the last section of this paper. 
3. Proper intersection effective Nullstellensatz. In what follows, $\mathbb{E}$ denotes the unit disc in $\mathbb{C}$. The first result comes from observing that one can easily prove Lemma 1.1 from [PT] in the c-holomorphic setting:

Lemma 3.1. Let $X$ be a pure $k$-dimensional analytic set in an open set $\Omega \subset \mathbb{C}^{m}$ and let $\varphi=\left(\varphi_{1}, \ldots, \varphi_{k}\right): X \rightarrow \mathbb{E}^{k}$ be a proper c-holomorphic mapping. Denote by $d$ its multiplicity and fix $l \in\{1, \ldots, k\}$. Then for each $g \in \mathcal{O}_{\mathrm{c}}(X)$ such that $g^{-1}(0) \supset\left\{x \in X \mid \varphi_{1}(x)=\cdots=\varphi_{l}(x)=0\right\}$ there exist $h_{1}, \ldots, h_{l} \in \mathcal{O}_{\mathrm{c}}(X)$ such that $g^{d}=\sum_{j=1}^{l} h_{j} \varphi_{j}$.

Proof. We consider the set $\Gamma:=\left\{(\varphi(x), g(x)) \in \mathbb{E}^{k} \times \mathbb{C} \mid x \in X\right\}$. It is easy to see that it is a pure $k$-dimensional analytic set.

For the generic $w \in \mathbb{E}^{k}$ one has exactly $d$ distinct points in the fibre $\varphi^{-1}(w)=\left\{x_{1}, \ldots, x_{d}\right\}$. For such points $w$ which moreover do not lie in the analytic set $\varphi(\operatorname{Sng} X) \subsetneq \mathbb{E}^{k}$ we may define

$$
P(w, t):=\prod_{j=1}^{d}\left(t-g\left(x_{j}\right)\right)=t^{d}+a_{1}(w) t^{d-1}+\cdots+a_{d}(w),
$$

where $a_{j}(w)=(-1)^{j} \sum_{1 \leq i_{1}<\cdots<i_{j} \leq d} g\left(x_{i_{1}}\right) \cdots g\left(x_{i_{j}}\right)$ are obviously continuous. It is also clear that outside an analytic proper subset of $\mathbb{E}^{k}$ these coefficients are holomorphic, whence by Riemann's theorem they are holomorphic on $\mathbb{E}^{k}$. Furthermore, $P^{-1}(0)=\Gamma$ and

$$
\Gamma \cap\left(\{0\}^{l} \times \mathbb{E}^{k-l} \times \mathbb{C}\right)=\{0\}^{l} \times \mathbb{E}^{k-l} \times\{0\},
$$

whence $\left.a_{j}\right|_{\left(\{0\}^{l} \times \mathbb{E}^{k-l}\right)} \equiv 0$ for all $j$. This in turn obviously means that for all $j, a_{j}(w)=y_{1} a_{j, 1}(w)+\cdots+y_{l} a_{j, l}(w)$, where $w=(y, z) \in \mathbb{E}^{l} \times \mathbb{E}^{k-l}$ and $a_{j, s} \in \mathcal{O}\left(\mathbb{E}^{k}\right), s=1, \ldots, l$.

Finally, we obtain the result sought for from $P(\varphi(x), g(x))=0$.

We now turn to generalizing the effective Nullstellensatz to the c-holomorphic case using Lemma 3.1 and the methods of [PT].

Let $A$ be a pure $k$-dimensional analytic set in an open set $\Omega \subset \mathbb{C}^{m}$ and let $f_{1}, \ldots, f_{n} \in \mathcal{O}_{\mathrm{c}}(A)$ be such that for $f=\left(f_{1}, \ldots, f_{n}\right)$ the set $f^{-1}(0)$ is of pure dimension $k-n$ (then the intersection of $\Gamma_{f}$ with $\Omega \times\{0\}^{n}$ is proper in $\Omega \times \mathbb{C}^{n}$ ). We denote by $Z_{f}$ the (Draper) cycle defined by

$$
Z_{f}=\Gamma_{f} \cdot\left(\Omega \times\{0\}^{n}\right) .
$$

THEOREM 3.2. In the above setting, for any $g \in \mathcal{O}_{\mathrm{c}}(A)$ such that $g^{-1}(0)$ $\supset f^{-1}(0)$ and for all $a \in A$, there exists a neighbourhood $U \ni a$ in $\Omega$ and functions $h_{1}, \ldots, h_{n} \in \mathcal{O}_{\mathrm{c}}(U \cap A)$ such that

$$
g^{\operatorname{deg}_{a} Z_{f}}=\sum_{j=1}^{n} h_{j} f_{j} \quad \text { on } U \cap A .
$$


Proof. Fix $a \in A$. For convenience we may assume that $a=0$. Suppose also that the coordinates in $\mathbb{C}^{m}$ are chosen in such a way that $\{0\}^{k-n} \times \mathbb{C}^{m-(k-n)}$ realizes $\operatorname{deg}_{0} Z_{f}$, i.e. their proper isolated intersection at zero has multiplicity $d:=\operatorname{deg}_{0} Z_{f}$. In particular 0 is isolated in the fibre $\varphi^{-1}(0)$, where $\varphi: A \ni x \mapsto\left(f(x), x_{1}, \ldots, x_{k-n}\right) \in \mathbb{C}^{k}$ is c-holomorphic. Thus, for some neighbourhood $V=U \cap A \ni 0$, the restriction $\left.\varphi\right|_{V}: V \rightarrow \varphi(V)$ is proper, $\varphi^{-1}(0) \cap V=\{0\}$ and obviously $\varphi(V)$ is open. We may assume that $\varphi(V)=\mathbb{E}^{k}$. By Lemma 3.1 it suffices to check that the multiplicity of $\varphi$ at zero is equal to $d$.

It is easy to check that the multiplicity of $\varphi$ is equal to the multiplicity (at zero) of the projection

$$
\pi: U \times \mathbb{C}^{n} \ni\left(x_{1}, \ldots, x_{m}, y_{1}, \ldots, y_{n}\right) \mapsto\left(y_{1}, \ldots, y_{n}, x_{1}, \ldots, x_{k-n}\right) \in \mathbb{C}^{k}
$$

when restricted to the set $\Gamma:=\Gamma_{f} \cap\left(U \times \mathbb{C}^{n}\right)$. But this is the multiplicity of proper isolated intersection of $\Gamma$ with $\pi^{-1}(0)$ at zero.

It remains to observe that by [TW, Theorem 2.2] we have

$$
\begin{aligned}
\Gamma \cdot \pi^{-1}(0) & =\left(\Gamma \cdot\left(U \times\{0\}^{n}\right)\right) \cdot U \times\{0\}^{n}\left(\{0\}^{k-n} \times \mathbb{C}^{m-(k-n)}\right) \\
& =Z_{f} \cdot\left(\{0\}^{k-n} \times \mathbb{C}^{m-(k-n)}\right)=d\{0\} .
\end{aligned}
$$

EXAMPLE 3.3. The coefficients $h_{j}$ in the Nullstellensatz formula of Theorem 3.2 may well be strictly c-holomorphic (i.e. have no holomorphic extension onto any neighbourhood of $a$ in $\left.\mathbb{C}^{m}\right)$. To see this consider the following simple example. Let $A:=\left\{(x, y) \in \mathbb{C}^{2} \mid y^{2}=x^{3}\right\}, f(x, y)=y / x$ for $(x, y) \in A \backslash\{(0,0)\}$ and $f(0,0)=0$. Then $f \in \mathcal{O}_{\mathrm{c}}(A)$ and it cannot be the restriction of a holomorphic function in any neighbourhood of zero in $\mathbb{C}^{2}$ (see $[\mathrm{Wh}]$ or $[\mathrm{D}])$. We compute (see e.g. [D])

$$
Z_{f}=i\left(\Gamma_{f} \cdot\left(\mathbb{C}^{2} \times\{0\}\right) ; 0\right)\{0\}^{2}=m_{0}(f)\{0\}^{2}=\operatorname{ord}_{0}(f \circ \gamma)\{0\}^{2},
$$

where $m_{0}(f)$ is the geometric multiplicity of $f$ at zero and $\gamma(t)=\left(t^{2}, t^{3}\right)$ is the Puiseux parametrization of $A$. Thus $\operatorname{deg}_{0} Z_{f}=1$.

Now take $g(x, y)=x$ restricted to $A$. Then $g=h f$ on $A$ with $h=f$ and $h$ is uniquely determined since one can just divide $g / f\left(\operatorname{as}_{\operatorname{ord}}(g \circ \gamma) \geq\right.$ $\left.\operatorname{ord}_{0}(f \circ \gamma)\right)$ to obtain $h$.

4. Isolated improper intersection effective Nullstellensatz. Let $A$ and $f$ be as in the previous section but suppose now $f^{-1}(0)=\{0\}^{m} \subset A$ (in particular $n \geq k)$. The intersection $\Gamma_{f} \cap\left(\Omega \times\{0\}^{n}\right)$ may not be proper (unless $n=k)$. In this case $\operatorname{deg}_{0} Z_{f}=i\left(\Gamma_{f} \cdot\left(\Omega \times\{0\}^{n}\right) ; 0\right)$, where the intersection multiplicity is calculated according to [ATW]. Thus in fact $\operatorname{deg}_{0} Z_{f}=m_{0}(f)$ where the latter is the geometric multiplicity of $f$ at zero. The following theorem generalizes to c-holomorphic functions one of the results of $[\mathrm{Cg}]$. 
THEOREM 4.1. In the above setting, for any $g \in \mathcal{O}_{\mathrm{c}}(A)$ such that $g(0)=0$, there is a neighbourhood $U$ of zero and functions $h_{1}, \ldots, h_{n} \in$ $\mathcal{O}_{\mathrm{c}}(U \cap A)$ such that

$$
g^{m_{0}(f)}=\sum_{j=1}^{n} h_{j} f_{j} \quad \text { on } U \cap A .
$$

Proof. As in the proof of Theorem (2.6) from [D] we may assume that $\Omega$ is small enough to have $f(A)$ analytic in a neighbourhood of $0 \in \mathbb{C}^{n}$. Fix any linear mapping $\Phi \in \mathrm{L}\left(\mathbb{C}^{m}, \mathbb{C}^{k}\right)$ of rank $k$ satisfying $\operatorname{Ker} \Phi \cap C_{0}(f(A))=\{0\}^{n}$.

Take any $g \in \mathcal{O}_{\mathrm{c}}(A)$ vanishing at zero. Then by Theorem 3.2 applied to the mapping $\Phi \circ f$, we find a neighbourhood $U$ of zero and c-holomorphic functions $\widetilde{h}_{j}$ in $U \cap A$ such that

$$
g^{\operatorname{deg}_{a} Z_{\Phi \circ f}}=\sum_{j=1}^{n} \widetilde{h}_{j} \cdot(\Phi \circ f)_{j} \quad \text { on } U \cap A .
$$

We have $\operatorname{deg}_{a} Z_{\Phi \circ f}=i\left(\Gamma_{\Phi \circ f} \cdot\left(\Omega \times\{0\}^{k}\right) ; 0\right)$ and we check exactly as in the proof of Theorem (2.6) from [D] (see also [S]) that the latter is equal to $i\left(\Gamma_{f} \cdot\left(\Omega \times\{0\}^{n}\right) ; 0\right)$, which is $m_{0}(f)$.

It remains to observe that since $(\Phi \circ f)_{j}=\Phi_{j} \circ f$ and $\Phi_{j}(y)=\sum_{\iota=1}^{n} \alpha_{\iota}^{j} y_{\iota}$, where $\alpha_{\iota}^{j} \in \mathbb{C}$, we obtain $\Phi_{j} \circ f=\sum_{\iota}^{n} \alpha_{\iota}^{j} f_{\iota}$ and so we may put

$$
h_{j}:=\sum_{\kappa=1}^{n} \alpha_{j}^{\kappa} \widetilde{h}_{\kappa} \in \mathcal{O}_{\mathrm{c}}(U \cap A)
$$

in order to get the assertion.

5. On the dimension. We end this paper with a useful remark (answering a question of Piotr Tworzewski) which is not at all obvious at first glance. It concerns the proper intersection of analytic sets and may be treated as a commentary to $[\mathrm{D}]$.

Proposition 5.1. Let $A$ be a pure $k$-dimensional analytic set in an open set $\Omega \subset \mathbb{C}^{m}$ and let $f_{1}, \ldots, f_{n} \in \mathcal{O}_{\mathrm{c}}(A)$ be such that for $f=\left(f_{1}, \ldots, f_{n}\right)$ the set $f^{-1}(0)$ has pure dimension $k-n$. Then for all $l \in\{1, \ldots, n\}$ the set $\bigcap_{j=1}^{l} f_{j}^{-1}(0)$ has pure dimension $k-l$.

To prove this we shall need two lemmata:

LeMmA 5.2. Let $A$ be an irreducible analytic set in an open set $\Omega \subset \mathbb{C}^{m}$ and let $f \in \mathcal{O}_{\mathrm{c}}(A)$. If there is an open set $U$ such that $f \equiv 0$ on $U \cap A \neq \emptyset$, then $f \equiv 0$.

Proof. This follows easily from the identity principle. Indeed, since Reg $A$ is a connected manifold and $U \cap \operatorname{Reg} A$ is a non-empty and open subset in it on which the holomorphic function $\left.f\right|_{\operatorname{Reg} A}$ vanishes, we have $f \equiv 0$ on 
$\operatorname{Reg} A$. By the continuity of $f$ and the density of regular points the assertion follows.

Using this lemma we obtain

LEMMA 5.3. Let $A$ be an irreducible $k$-dimensional analytic set in an open set $\Omega \subset \mathbb{C}^{m}$ and let $f \in \mathcal{O}_{\mathrm{c}}(A)$ be non-constant. Then $f^{-1}(0)$ is either empty or has pure dimension $k-1$.

Proof. If $f^{-1}(0)$ is not empty, then since

$$
f^{-1}(0) \times\{0\}=\Gamma_{f} \cap(\Omega \times\{0\}) \neq A \times\{0\},
$$

one clearly has at each point $a \in f^{-1}(0)$ the inequalities

$$
k-1=k+m-(m+1) \leq \operatorname{dim}_{a} f^{-1}(0)<k
$$

and so $\operatorname{dim}_{a} f^{-1}(0)=k-1$ (remember $A$ is irreducible).

REMARK 5.4. It is worth noting that the above result does not hold in the weakly holomorphic case (the zero-set is then replaced by the intersection of the closure of the graph with $A \times\{0\})$. To see this consider the function $g$ from Example 2.3.

Proof of Proposition 5.1. In view of the upper semicontinuity of the dimension it suffices to consider the problem locally. Therefore we restrict ourselves to a point $a \in \bigcap_{j=1}^{n-1} f_{j}^{-1}(0)$ and we may assume that $a=0$.

From now on we consider the $f_{j}$ as function germs at zero. We put $Z_{j}:=$ $\left\{f_{j}=0\right\}$ (as germs at zero).

Let $A_{0}=A_{1} \cup \cdots \cup A_{r}$ be the decomposition of the germ $A_{0}$ into irreducible components. If $\left.f_{1}\right|_{A_{j}} \neq \equiv 0$ for all $j$ then $Z_{1}$ has pure dimension $k-1$ by Lemma 5.3. On the other hand, if $\left.f_{1}\right|_{A_{j}} \equiv 0$ for some $j$, then $Z_{1} \cap A_{j}=A_{j}$ and the dimension at zero of the zero-set of $\left.f_{2}\right|_{A_{j}}$ is not less than $k-1$. Thus, the zero-set of $\left.f_{3}\right|_{2} \cap A_{j}$ has dimension at least $k-2$ (since the irreducible components at zero of the analytic germ $Z_{2} \cap A_{j}$ have dimension not smaller than $k-1)$.

Continuing this procedure we conclude that the dimension of the intersection germ $Z_{n} \cap \cdots \cap Z_{2} \cap A_{j}=Z_{n} \cap \cdots \cap Z_{1} \cap A_{j}$ must be at least $k-n+1$, contrary to our assumptions. Thus $Z_{1}$ is of dimension $k-1$, i.e. $Z_{1} \cap A_{j} \subsetneq A_{j}$ for all $j$.

We now repeat the argument for $\left.f_{2}\right|_{Z_{1}}$ and the irreducible components of $Z_{1}$ concluding that $Z_{1} \cap Z_{2}$ has dimension $k-2$.

\section{References}

[ATW] R. Achilles, P. Tworzewski and T. Winiarski, On improper isolated intersection in complex analytic geometry, Ann. Polon. Math. 51 (1990), 21-36. 
[Cg] E. Cygan, Nullstellensatz and cycles of zeroes of holomorphic mappings, ibid. 78 (2002), 181-191.

[D] M. P. Denkowski, The Eojasiewicz exponent of c-holomorphic mappings, ibid. 87 (2005), 63-81.

[Dr] R. N. Draper, Intersection theory in analytic geometry, Math. Ann. 180 (1969), 175-204.

[Ł] S. Łojasiewicz, Introduction to Complex Analytic Geometry, Birkhäuser, Basel, 1991.

[PT] A. Płoski and P. Tworzewski, Effective Nullstellensatz on analytic and algebraic varieties, Bull. Polish Acad. Sci. Math. 46 (1998), 31-38.

[R] R. Remmert, Projektionen analytischer Mengen, Math. Ann. 130 (1956), 410441.

[S] S. Spodzieja, Multiplicity and the Eojasiewicz exponent, Ann. Polon. Math. 73 (2000), 257-267.

[T] P. Tworzewski, Intersection theory in complex analytic geometry, ibid. 62 (1995), $177-191$.

[TW] P. Tworzewski and T. Winiarski, Cycles of zeroes of holomorphic mappings, Bull. Polish Acad. Sci. Math. 37 (1986), 95-101.

[Wh] H. Whitney, Complex Analytic Varieties, Addison-Wesley, 1972.

Institute of Mathematics

Jagiellonian University

Reymonta 4

30-059 Kraków, Poland

E-mail: denkowsk@im.uj.edu.pl
Institut de Mathématiques de Bordeaux Université Bordeaux 1 351, cours de la Libération 33405 Talence, France E-mail: denkowsk@math.u-bordeaux1.fr

Received 13.1.2006

and in final form 7.1.2007 